\title{
Primary pure carcinoid tumors of the testis: Clinicopathological and immunophenotypical characteristics of 11 cases
}

\author{
CHANGLI LU ${ }^{1}$, ZHANG ZHANG ${ }^{1}$, YONG JIANG ${ }^{1}$, ZHIRONG YANG ${ }^{2}$, QUNPEI YANG ${ }^{1}$, \\ DIANYING LIAO ${ }^{1}$ and $\mathrm{HONG} \mathrm{BU}^{1}$ \\ ${ }^{1}$ Department of Pathology, West China Hospital, Sichuan University, Chengdu, Sichuan 610041; \\ ${ }^{2}$ Department of Pathology, People's Hospital of Ya'an City, Ya'an, Sichuan 625000, P.R. China
}

Received May 4, 2014; Accepted February 17, 2015

DOI: $10.3892 / 01.2015 .3046$

\begin{abstract}
Primary pure carcinoid tumors of the testis (рPCTT) are rare, and there are only a limited number of studies available. In the present study we described the clinicopathological and immunophenotypical characteristics of 11 cases from our institution between 1978 and 2014, and reported our experiences of the diagnosis and treatment of these patients. The patients ranged in age from 26 to 68 years old, with a median age of 48 years. One patient $(9 \%)$ was classified as pT2 and 10 (91\%) were pT1. Histologically, 7 cases were diagnosed as classical carcinoid tumors, while the other 4 cases were identified as atypical carcinoid tumors. The most common growth pattern was a mixed insular, acinar, rosetted, solid and trabecular pattern. Immunohistochemical staining revealed positive expression of neuron-specific enolase in all cases, and $\mathrm{CgA}$, Syn and CD56 markers in $8(72.7 \%), 10(90.9 \%)$ and 9 cases $(81.7 \%)$, respectively. In addition to radical orchiectomy, 9 patients $(81.7 \%)$ received a combined modality of treatment. Follow-up data were available for 8 patients. Seven were alive at the last follow-up without recurrence, and one patient succumbed to cerebral hemorrhage 7 years after surgery. In summary, localized pPCTT is a rare disease with an indolent clinical course. When a testicular carcinoid tumor is identified, a metastasis or an intestinal primary tumor should be excluded, particularly when the testicular tumor is large. A tumor size $\leq 6.0 \mathrm{~cm}$ and the histological appearance had little relation with metastatic behavior.
\end{abstract}

Correspondence to: Dr Changli Lu, Department of Pathology, West China Hospital, Sichuan University, 37 Guoxue Xiang, Chengdu, Sichuan 610041, P.R. China

E-mail: 13408587426@163.com

Key words: carcinoid tumor, atypical, testicle, pure, metastasis

\section{Introduction}

Carcinoid tumors have been reported in a wide range of organs, but they most commonly involve the gastrointestinal and bronchopulmonary systems. Carcinoid tumors are rare in the testis and comprise $<1 \%$ of all testicular neoplasms (1). In a review of 13,715 carcinoid tumors, 9176 cases $(66.9 \%)$ occurred in the gastrointestinal tract, whereas 113 cases $(0.8 \%)$ occurred in the ovary, with only 8 cases $(0.06 \%)$ observed in the testicle (2). Unlike ovarian carcinoid tumors, testicular carcinoid tumors are rarely associated (in $<3 \%$ of cases) with carcinoid syndrome (3).

Testicular carcinoid tumors can be divided into three subgroups: primary pure testicular carcinoid tumors, carcinoid tumors associated with teratoma ( $20 \%$ of cases), and carcinoid metastasis to the testis ( $\sim 9 \%$ of cases). Cases have been reported with patients ranging in age from ten to eighty-three years, which is older than that reported with most primary germ cell tumors (20-40 years) (1).

The first published case of a testicular carcinoid tumor was observed as an element of a benign cystic teratoma in 1954 (4). Since then, around 150 cases of carcinoid tumors in the testis have been reported (5-11). Data on ethnicity and geographical distribution have revealed that the majority of carcinoid tumors occurred in Europe or the United States. Our extensive literature search yielded fewer than 30 cases from Asia $(3,6,12)$. In this study, we investigated the clinicopathological and immunophenotypical characteristics of primary pure carcinoid tumors of the testis (pPCTT) in 11 Chinese patients, and performed a comparison with the cases reported in the literature.

\section{Materials and methods}

Clinical information. This study was approved by the ethics committee of West China Hospital in Sichuan University, Chengdu, China. Between January 1978 and January 2014, 688 male patients with testicular neoplasms (including germ cell tumors, sex cord/gonadal stromal tumors, lymphomas and leukemia) underwent surgical resections in our medical center. Only 7 cases $(1.02 \%)$ were pathologically diagnosed 
Table I. Clinical and pathological features of 11 male patients with primary pure carcinoid tumors of the testis.

\begin{tabular}{|c|c|c|c|c|c|c|c|c|}
\hline $\begin{array}{l}\text { Case } \\
\text { no. }\end{array}$ & $\begin{array}{c}\text { Age } \\
\text { (years) }\end{array}$ & Clinical history & $\begin{array}{l}\text { Duration }^{\mathrm{a}} \\
\text { (months) }\end{array}$ & $\begin{array}{l}\text { Location } \\
\text { (testicle) }\end{array}$ & $\begin{array}{c}\text { Tumor } \\
\text { size }(\mathrm{cm})\end{array}$ & $\begin{array}{c}\text { Histological } \\
\text { grade }\end{array}$ & $\begin{array}{l}\text { Mitosis } \\
\text { (/10 HPF) }\end{array}$ & $\begin{array}{l}\text { Follow-up } \\
\text { (years) }\end{array}$ \\
\hline 1 & 48 & Painless mass & 4 & Right & 2.5 & Classical & $\leq 1$ & Lost to follow-up \\
\hline 2 & 26 & Painless mass & 36 & Right & 5.2 & Classical & $\leq 1$ & Lost to follow-up \\
\hline 3 & 31 & Painless mass & 24 & Right & 2.0 & Classical & $\leq 1$ & Lost to follow-up \\
\hline 4 & 49 & Swelling and pain & 120 & Right & 2.5 & Classical & $\leq 1$ & 7 years, died $^{\mathrm{b}}$ \\
\hline 5 & 36 & Swelling and pain & 1 & Right & 3.1 & Classical & $\leq 1$ & 9 years, alive \\
\hline 6 & 59 & Swelling and discomfort & 0.25 & Right & 4.0 & Classical & $\leq 1$ & 5 years, alive \\
\hline 7 & 66 & Painless mass & 24 & Right & 6.0 & Classical & $\leq 1$ & 4 years, alive \\
\hline 8 & 68 & Swelling and discomfort & 0.5 & Right & 3.2 & Atypical & $2-4$ & 3 years, alive \\
\hline 9 & 41 & Painless mass & 12 & Left & 3.6 & Atypical & $2-3$ & 3 years, alive \\
\hline 10 & 30 & Painless mass & 12 & Right & 2.5 & Atypical & $2-4$ & 3 years, alive \\
\hline 11 & 61 & Painless mass & 12 & Left & 5.0 & Atypical & $2-5$ & 5 years, alive \\
\hline
\end{tabular}

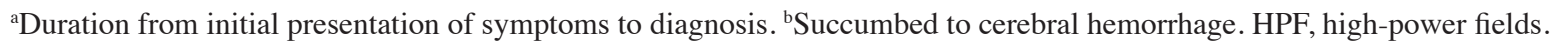

as primary pure carcinoid tumors of the testis. In addition, 6 pPCTT patients from other hospitals were sent to our hospital for consultation and further evaluation. Two of the 13 patients were excluded following further evaluation due to insufficient materials or incomplete clinical data. Finally, 11 male patients were enrolled in the study. All 11 patients underwent unilateral radical orchiectomy.

The clinical features of the 11 patients including clinical course and follow-up data were compiled. Furthermore, the patients had staging evaluations that consisted of thorough physical examinations by pre-operative imaging studies including computed tomography (CT) scans of the chest, abdomen and pelvis, duodenal fibroscopy and colonoscopy as well as somatostatin receptor scintigraphy. In addition, the serum levels of three tumor markers $\beta$ human chorionic gonadotrophin ( $\beta$-HCG), $\alpha$-fetoprotein (AFP) and lactate dehydrogenase $(\mathrm{LDH})$ were also determined in the patients.

Histological examination. Surgical resected specimens of testicular tumors from the 11 patients were fixed with $10 \%$ formalin, routinely embedded in paraffin, and the tissue sections were stained with hematoxylin and eosin (H\&E). The H\&E sections were independently diagnosed by two experienced pathologists. Histological diagnosis of the tumors was based on the World Health Organization criteria for the classification of carcinoid tumors in the pancreas (13).

Immunophenotypical analysis. Primary antibodies used in this study were rabbit polyclonal antibodies to adrenocorticotropin hormone (ACTH), gastrin, glucagon, pancreatic polypeptide (PP) and somatostatin (Maxim Biotech, San Francisco, CA, USA); chromogranin A (CgA; Zymed Laboratories, San Francisco, CA, USA); CD117 (DakoCytomation, Glostrup, Denmark); mouse monoclonal antibodies to inhibin- $\alpha$, human melanoma black 45, neuron-specific enolase (NSE), epithelial membrane antigen, placental alkaline phosphatase (PLAP), thyroid transcription factor 1 (TTF-1) and Syn (DakoCytomation); Ki67, CDX-2 and S-100 (Maxim Biotech); a broad-spectrum cytokeratin (CK) cocktail and CD56 (Zeta Corporation, Sierra Madre, CA, USA) and melanoma antigen recognized by $\mathrm{T}$ cells 1 (Zymed Laboratories). The Envision kit (DakoCytomation) was used for immunohistochemical staining according to the manufacturer's instructions.

\section{Results}

Clinicopathological features. The clinical characteristics of the 11 male patients with pPCTT are presented in Table I. The median age of the patients was 47 years (range, 26-68 years). The mean time from the initial presentation of symptoms to diagnosis was 22.3 months. Seven patients $(63.6 \%)$ noted a painless mass in one testicle, while 4 patients (36.4\%) experienced testicular swelling and pain or discomfort. Case 5 had noted his right testicle to be larger than the left since his childhood, but did not pay close attention until he experienced a dull scrotal pain for one month. Case 3 was identified as having a mass in his right testicle during skin degerming prior to surgery for acute appendicitis.

Among the 11 patients, $9(81.8 \%)$ had the tumor in the right testicle, while $2(18.2 \%)$ had the tumor in the left testicle, as confirmed by ultrasound scan (Fig. 1). Case 1 had right inguinal lymphadenopathy. For all 11 patients, no visible metastasis was observed in the liver, retroperitoneum or other organs by the imaging studies. None of the patients had associated carcinoid syndrome. Furthermore, the serum levels of the three tumor markers ( $\beta$-HCG, AFP and LDH) were in the normal range. Finally, one (9\%) patient was classified as pT2 and $10(91 \%)$ as pT1. All were N0M0 and without carcinoid syndrome at initial diagnosis according to pathological tumornode-metastasis staging.

Pathological findings. The gross tumor size ranged from 2.0 to $6.0 \mathrm{~cm}$ (mean, $3.6 \mathrm{~cm}$ ) in diameter in the largest dimension (Table I). In all 11 cases the tumors were well demarcated from the surrounding parenchyma but not encapsulated. They were nodular tumors with solid, homogeneous, yellow-tan 
A

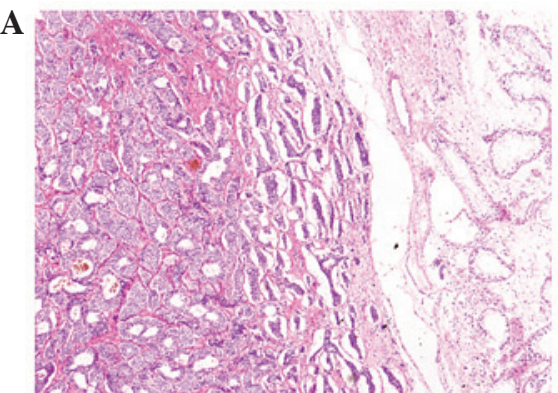

C

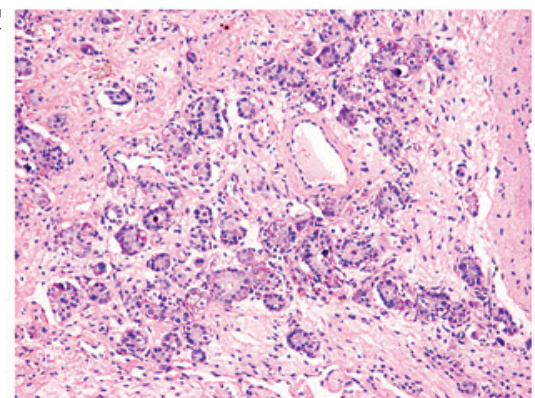

E

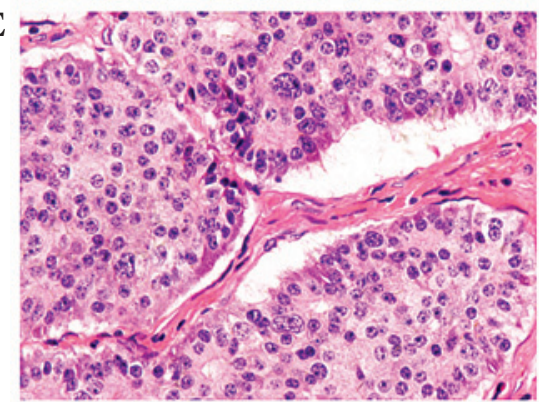

G

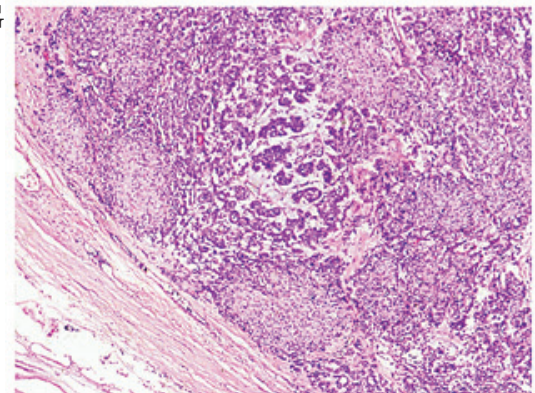

B

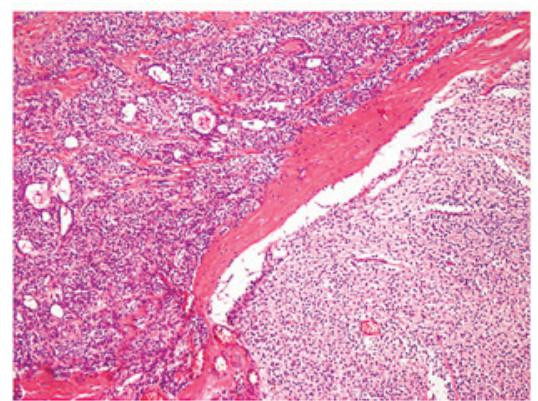

D

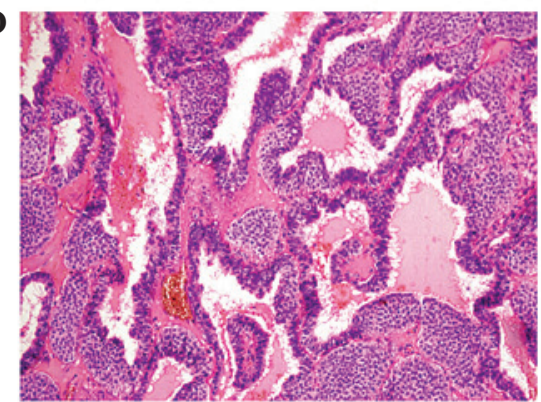

F

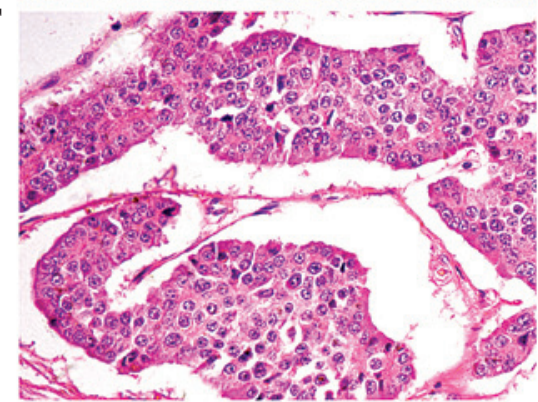

H

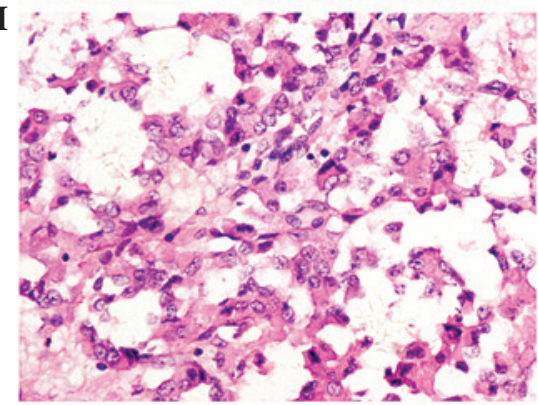

Figure 1. Hematoxylin and eosin-stained histological images of pure carcinoid tumors and surrounding testicular parenchyma. (A) Carcinoid tumor with a mixed insular, acinar, rosetted, solid and trabecular pattern separated by delicate fibroconnective stroma (magnification, x20). (B) Diffuse solid growth pattern (right bottom) with a desmoplastic stromal reaction and mixed with acinar and trabecular patterns from case 10 (magnification, x10). (C) Neoplastic cells demonstrating follicular or pseudoglandular patterns filled with condensed secretory fluid stimulating the psammoma body (magnification, $\mathrm{x} 50$ ). (D) Neoplastic cells demonstrating follicular or pseudoglandular patterns filled with eosinophilic secretory fluid, and erythrocytes resembling colloid goiter (magnification, x20). (E) Neoplastic cells are relatively homogeneous, medium in size, have oval to round nuclei, with prominent chromatin clumping along the nuclear envelope, inconspicuous nucleoli and a scant-to-moderate amount of eosinophilic granular cytoplasm (magnification, $\mathrm{x} 40$ ). (F) Focal nuclear pleomorphism and apoptosis are present in the neoplastic cells (magnification, $\mathrm{x} 40$ ). (G) Diffuse solid growth pattern with a mucoid stromal reaction from case 11 (magnification, x20). (H) Pathological mitotic figures are observed in an atypical carcinoid tumor (magnification, x40).

cut surfaces. The residual testicular parenchyma surrounding the tumors was compressed and atrophic. Case 1 had slightly enlarged lymph nodes in the right inguinal region, which demonstrated reactive hypertension in the biopsy specimens.

Histologically, the carcinoid tumors demonstrated multiple growth patterns at low-power magnification. The most common type was a mixed insular, acinar, rosetted, solid and trabecular pattern separated by delicate fibroconnective stroma (Fig. 1A and B). Occasionally, the neoplastic cells were arranged in follicular or pseudoglandular patterns filled with condensed secretory fluid, stimulating the psammoma body (Fig. 1C), and erythrocytes resembled colloid goiter (Fig. 1D). At higher power magnification, the neoplastic cells were relatively homogeneous, medium in size, had oval to round nuclei with prominent chromatin clumping along the nuclear envelope, inconspicuous nucleoli and a scant-tomoderate amount of eosinophilic granular cytoplasm. Focal nuclear pleomorphism was noted, occasionally with giant cells (Fig. 1E and F). Two cases (cases 10 and 11) demonstrated a diffuse solid growth pattern with a desmoplastic or mucoid stromal reaction in certain areas (Fig. 1B right bottom and $\mathrm{G})$. 


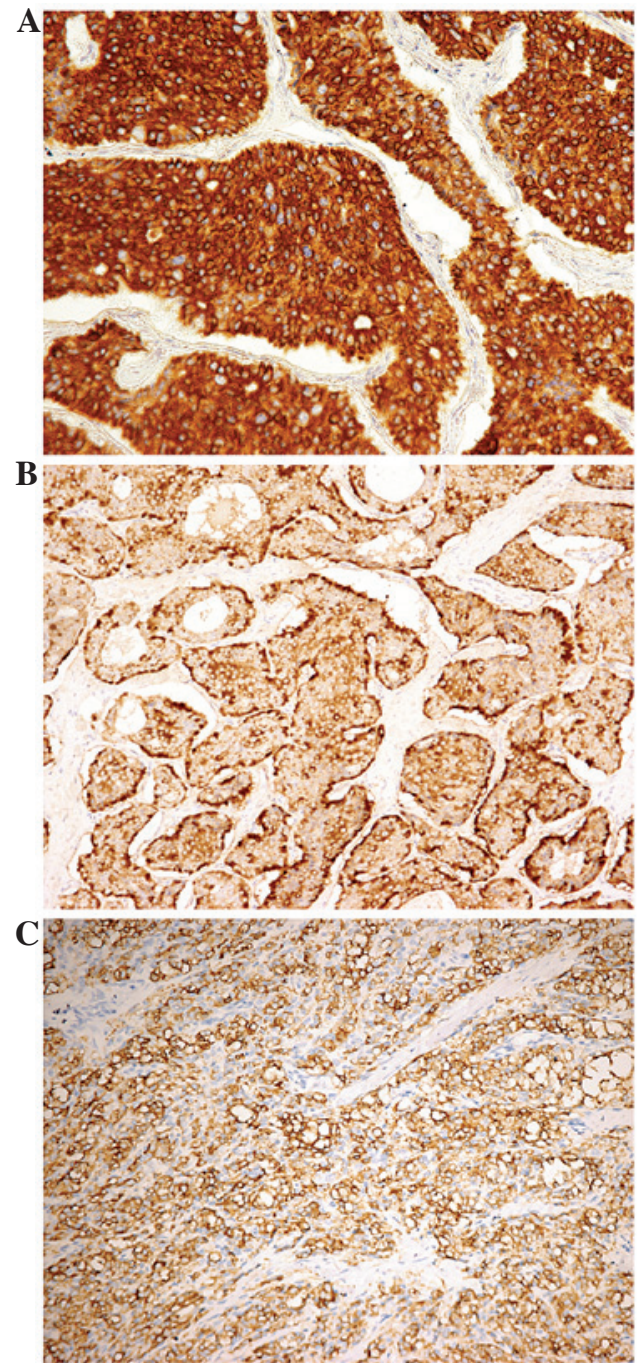

Figure 2. Immunohistochemically stained histological images of pure carcinoid tumors and surrounding testicular parenchyma. (A) Neoplastic cells are positive for cytokeratin (magnification, x20). (B) Neoplastic cells are positive for chromogranin A (magnification, x100). (C) Neoplastic cells are positive for Syn (magnification, x20).

In the 11 patients, 7 cases (cases 1 to 7) were diagnosed as classical carcinoid tumors with $\leq 1$ mitotic figure per 10 high-power fields (HPF), while the other 4 cases (cases 8 to 11) were identified as atypical carcinoid tumors with 2-5 mitoses per 10 HPF (Table I, Fig. 2H). Focal inflammatory cells (mainly lymphocytes) were only observed in 3 cases. Necrosis and vascular invasion by tumor cells were not observed in any of the 11 cases. Neither teratomatous elements nor intratubular germ cell neoplasia were observed in any cases. The tunica albuginea was thickened by fibrosis, while the epididymis and spermatic cords did not significantly change.

Immunohistochemical features. All neoplastic cells were strong and diffusely positive for NSE staining. CK antigen (Fig. 2A) was expressed in 9 cases (81.8\%), while CgA (Fig. 2B) and Syn (Fig. 2C) antigens were expressed in 8 (72.7\%) and 10 cases (90.9\%), respectively. In 9 of the 11 cases (81.8\%), the neoplastic cells were positive for CD56. Ki67 labeling was performed in 10 cases, which revealed that $\leq 2 \%$ of the tumor cells were typical carcinoid and $3-7 \%$ of the tumor cells were atypical carcinoid. None of the tumors reacted with CD117, PLAP, AFP, ACTH, gastrin, glucagon, PP or somatostatin. CDX-2 and TTF-1 were negative in all cases. An additional five markers (HCG, $\alpha$-inhibin, calretinin, MART1 and HBME1) were used for 2 patients (cases 10 and 11), in whom the tumors demonstrated negative staining for these five markers.

Follow-up results. In addition to radical orchiectomy, 9 patients $(81.7 \%)$ received a combined modality of treatment (radiotherapy, chemotherapy or both). Follow-up data were available for 8 patients (72.7\%). The mean follow-up time was 4.9 years, ranging from 3 to 9 years (Table I). Seven patients were alive during the follow-up without recurrence or metastases, while one patient had a history of hypertension and succumbed to cerebral hemorrhage 7 years after the surgery.

\section{Discussion}

Testicular carcinoid tumors are rare, and only limited studies have been carried out in China. The incidences of primary testicular carcinoids in North America and Japan are lower than 1 and $0.2 \%$,respectively $(6,8)$. At the West China Hospital, 688 male patients with testicular neoplasms underwent surgical resections between 1978 and 2013. Primary testicular carcinoid tumors accounted for $1.02 \%$ of these, which is similar to the reported incidence in North America.

Clinically, our cases presented with symptoms similar to those described in the literature. Stroosma and Delaere (1) reviewed 62 cases of testicular carcinoids reported from 1930 to 2006 all over the world. The most common presenting symptom was a painless scrotal mass in 35/62 of patients (79\%), which is slightly higher than the 7/11 (63.6\%) noted in our series. Since the symptoms are not specific and onset is insidious, a testicular carcinoid tumor is often not diagnosed early and not recognized until the physical examination prior to surgery for other diseases. In our 11 cases the longest duration from the initial presentation of symptoms to diagnosis was 10 years. The peak age was older than that observed with most primary germ cell tumors.

Carcinoid syndrome tumors are associated with 1.1-3.1\% of patients with primary testicular carcinoid tumors (3). Carcinoid syndrome is more common in carcinoid tumors with metastasis $(3,14,15)$. It has been reported that $50 \%$ of carcinoid tumor patients with metastases had carcinoid syndrome, compared with $5.6 \%$ of patients without metastases (16). It is unclear whether the presence of carcinoid syndrome is a feature associated with a malignant course in the testis (17). The absence of hormone immunoreactivity in our series may explain the lack of carcinoid syndrome in the majority of testicular carcinoid tumor cases. Our case study confirms that carcinoid syndrome is uncommon in primary testicular carcinoid tumors without metastasis.

Although the rate of metastasis from an extra-testicular source is low, occasionally a gastrointestinal primary carcinoid may metastasize to the testicle. Therefore, during the differential diagnosis of testicular carcinoid, metastasis or gastrointestinal primary carcinoid must be considered. There are significant implications for survival, as metastatic carcinoids are usually part of a widely disseminated disease with a poorer clinical course (5). One case of testicular metastasis 
was reported 10 years after resection of an appendiceal carcinoid (18). It is of limited value for site-specific markers to distinguish metastatic from primary pPCTT. Nuclear CDX-2 immunoreactivity was mainly observed in midgut neuroendocrine tumors, in which the majority of cells demonstrated strong positivity $(19,20)$. TTF-1 was identified in respiratory epithelial cells, but has also been reported in other sites including the breast, and in poorly differentiated neuroendocrine carcinoma of the cervix (21). However, none of the cases in our series demonstrated nuclear expression of CDX-2 or TTF-1. Therefore, extensive medical examinations, including a 24-h urinary 5-HIAA test, chest X-ray, CT scan of the abdomen and pelvis, PET-CT scan and possibly a gastrointestinal contrast study should be performed to exclude the possibility of metastasis from a carcinoid tumor in another organ (11). However, the metastatic tumors are generally multiple nodules in the bilateral testis and the persistence of carcinoid syndrome following orchiectomy. Furthermore, primary carcinoids of the testis are usually associated with other teratomatous elements, and occasionally with seminoma (22). Therefore, thorough gross examination and sufficient sampling are strongly recommended to exclude other components.

It is still possible to misdiagnose a testicular carcinoid tumor, particularly an atypical carcinoid tumor. For example, six consultant cases in our series of 11 patients with pPCTT from other hospitals were initially diagnosed as Sertoli cell tumors, paraganglioma, granulosa cell tumor or embryonal carcinoma. Sertoli cell tumors arranged in a hollow or solid tubular formation may demonstrate a neuroendocrine-like pattern, but the cytoplasm is clear or lightly vacuolated. Sertoli cell tumors are positive for $\alpha$-inhibin but negative for neuroendocrine markers. The insular and trabecular patterns of granulosa cell tumors may be mistaken for a carcinoid tumor. The presence of nuclear grooves and the absence of neuroendocrine markers distinguish the granulosa cell tumor from a carcinoid tumor. Paraganglioma arranged in an organoid nesting pattern may resemble a carcinoid tumor. However, this is extremely rare in the testis, and lacks CK staining which is frequently positive in carcinoids. Undifferentiated carcinomas and poorly differentiated adenocarcinomas may resemble atypical carcinoids. These carcinomas have abundant mitotic figures and have frequently already extended beyond the testis at presentation. Notably, case 11 in our study was initially misinterpreted as Sertoli cell tumors which were negative for $\alpha$-inhibin, calretinin, HBME1, CD30, PLAP, CgA and Syn, but positive for CK. We recommended CD56 and NSE staining for further evaluation, and the neoplastic cells were strongly positive for CD56 and NSE markers. When CgA and Syn markers are sparse or negative in the poorly differentiated tumors, the positive reaction of CD56 and NSE may support the diagnosis of a carcinoid tumor, although these markers have poor specificity.

In general, primary testicular carcinoids have an excellent prognosis, with certain cases only incidentally observed at autopsy (8). Approximately $11 \%$ of primary testicular carcinoid tumors exhibit malignant behavior $(3,15)$. Zavala-Pompa et al revealed that larger tumors $(7.3 \mathrm{vs} .2 .9 \mathrm{~cm})$ predicted increased metastatic potential, while tumor necrosis and local tumor invasion were not associated with adverse prognosis (17). In our study of 11 Chinese cases, the tumor sizes ranged from 2 to $6 \mathrm{~cm}$, and the majority $(81.8 \%)$ of the tumors were located in the right testicle. None of the patients had recurrence or metastases at presentation. Our case study suggests that the metastatic potential cannot be predicted by the histological appearance and tumor size $\leq 6.0 \mathrm{~cm}$.

Chemotherapy and radiotherapy are known to have minimal benefits for metastatic disease (23). Radical orchiectomy with close follow-up is the treatment of choice for organ-confined carcinoid (5,7). The longest reported interval between the presentation and diagnosis was 43 years for a case of uveal metastasis following resection of a bronchogenic carcinoid tumor (24). In our series of 11 patients, follow-up data were available for 8 patients $(72.7 \%)$ for 3-9 years. Although none of the 8 patients experienced recurrence or metastases, at least during the follow-up, the follow-up time may not be long enough for the natural course of carcinoid tumors. Follow-up should include physical examination and a 24-h urinary 5-HIAA test every 3 months for 1 year and then annually (25).

In conclusion, localized pPCTT is a rare disease with an indolent clinical course. When a testicular carcinoid tumor is identified, a multimodal approach should be taken to exclude an extra-testicular primary source, particularly when the testicular tumor is large. A tumor size $\leq 6.0 \mathrm{~cm}$ and the histological appearance had little relation with metastatic behavior. Follow-up must be extremely close due to the potential for delayed metastases.

\section{Acknowledgements}

The authors would like to thank Dr Ying Wu for editorial assistance in the preparation of the manuscript. This study was supported by the Ministry of Education PhD Program Fund (20120181110090).

\section{References}

1. Stroosma OB and Delaere KP: Carcinoid tumours of the testis. BJU Int 101: 1101-1105, 2008.

2. Modlin IM, Lye KD and Kidd M: A 5-decade analysis of 13,715 carcinoid tumors. Cancer 97: 934-959, 2003.

3. Hayashi T, Iida S, Taguchi J, et al: Primary carcinoid of the testis associated with carcinoid syndrome. Int J Urol 8: 522-524, 2001.

4. Simon HB, McDonald JR and Culp OS: Argentaffin tumor (carcinoid) occurring in a benign cystic teratoma of the testicle. J Urol 72: 892-894, 1954.

5. Wolf M, Wunderlich H, Hindermann W, et al: Primary carcinoid tumor of the testicle without metastases in combination with testicular atrophy and testosterone deficiency. Int Urol Nephrol 38: 625-628, 2006.

6. Kato N, Motoyama T, Kameda N, et al: Primary carcinoid tumor of the testis: Immunohistochemical, ultrastructural and FISH analysis with review of the literature. Pathol Int 53: 680-685, 2003.

7. Reyes A, Moran CA, Suster S, et al: Neuroendocrine carcinomas (carcinoid tumor) of the testis. A clinicopathologic and immunohistochemical study of ten cases. Am J Clin Pathol 120: 182-187, 2003.

8. Wang WP, Guo C, Berney DM, et al: Primary carcinoid tumors of the testis: a clinicopathologic study of 29 cases. Am J Surg Pathol 34: 519-524, 2010.

9. Ulbright TM and Young RH: Carcinoid tumor of the testis. Am J Clin Pathol 121: 297-298, 2004.

10. Talerman A, Gratama S, Miranda S, et al: Primary carcinoid tumor of the testis: case report, ultrastructure and review of the literature. Cancer 42: 2696-2706, 1978.

11. Neely D and Gray S: Primary carcinoid tumour of the testis. Ulster Med J 80: 79-81, 2011. 
12. Kim HJ, Cho MY, Park YN, et al: Primary carcinoid tumor of the testis: immunohistochemical, ultrastructural and DNA flow cytometric study of two cases. J Korean Med Sci 14: 57-62, 1999.

13. Oberg K, Akerstrom G, Rindi G, et al: Neuroendocrine gastroenteropancreatic tumours: ESMO clinical practice guidelines for diagnosis, treatment and follow-up. Ann Oncol 21 Suppl 5: v223-v227, 2010.

14. Son HY, Ra SW, Jeong JO, et al: Primary carcinoid tumor of the bilateral testis associated with carcinoid syndrome. Int J Urol 11: 1041-1043, 2004

15. Kaufman JJ and Waisman J: Primary carcinoid tumor of testis with metastasis. Urology 25: 534-536, 1985.

16. Thomas JC and Jones JS: Primary carcinoid tumor of the testis found at the time of elective sterilization. J Androl 25: 338-339, 2004.

17. Zavala-Pompa A, Ro JY, el-Naggar A, et al: Primary carcinoid tumor of testis. Immunohistochemical, ultrastructural and DNA flow cytometric study of three cases with a review of the literature. Cancer 72: 1726-1732, 1993.

18. Fucs M, Romero FR, Germanos de Castewm, et al: Testicular metastasis 10 years after resection of appendiceal carcinoid. Urology 65: 591, 2005.

19. Lin X, Saad RS, Luckasevic TM, et al: Diagnostic value of CDX-2 and TTF-1 expressions in separating metastatic neuroendocrine neoplasms of unknown origin. Appl Immunohistochem Mol Morphol 15: 407-414, 2007.
20. Chan ES, Alexander J, Swanson PE, et al: PDX-1, CDX-2, TTF-1 and CK7: a reliable immunohistochemical panel for pancreatic neuroendocrine neoplasms. Am J Surg Pathol 36: 737-743, 2012

21. McCluggage WG, Kennedy K and Busam KJ: An immunohistochemical study of cervical neuroendocrine carcinomas: Neoplasms that are commonly TTF1 positive and which may express CK20 and P63. Am J Surg Pathol 34: 525-532, 2010.

22. Hodzic J, Golka K and Schulze H: Primary testicular carcinoid. Med Sci Monit 10: CS46-CS48, 2004.

23. Modlin IM, Latich I, Kidd M, et al: Therapeutic options for gastrointestinal carcinoids. Clin Gastroenterol Hepatol 4: 526-547, 2006.

24. Kaiser ED, See RF, Rechdouni AK, et al: Uveal metastasis 43 years after resection of bronchogenic carcinoid. Br J Ophthalmol 86: 1191-1192, 2002.

25. Sutherland RS, Wettlaufer JN and Miller GJ: Primary carcinoid tumor of the testicle: a case report and management schema. J Urol 148: 880-882, 1992. 